\title{
Differential Effect of Hydrocortisone on Eosinophil and Neutrophil Proliferation
}

Barbara H. Bjornson, John M. Harvey, and Lynda Rose

William B. Castle Hematology Research Laboratory, Hematology/Oncology Section, Boston City Hospital; and Department of Medicine and Biostatistics Laboratory, Boston University School of Medicine, Boston, Massachusetts 02118

\begin{abstract}
Glucocorticosteroid therapy results in an increase in the number of circulating neutrophils and a decrease in the number of eosinophils. Utilizing the double layer soft agar technique, we examined the effect of physiologic to pharmacologic concentrations of hydrocortisone on the proliferation of human neutrophil progenitors and eosinophil progenitors from peripheral blood and bone marrow. When peripheral blood cultures were studied, eosinophil proliferation was inhibited in a dose-responsive fashion with $10^{-8}-10^{-5} \mathrm{M}$ hydrocortisone succinate, and comprised $49 \pm 4 \%$ of the colonies in control cultures and only $4 \pm 1 \%(P$ $<0.01)$ at pharmacologic levels of hydrocortisone $\left(10^{-5} \mathrm{M}\right)$. The number of neutrophil colonies, on the other hand, increased by $31 \%$ when $10^{-5} \mathrm{M}$ hydrocortisone was added to cultures. In order for corticosteroids to exert this effect, it was necessary to add them within $24 \mathrm{~h}$ of the initiation of culture. The effect of hydrocortisone on granulocyte proliferation could not be blocked by progesterone, a structurally analogous steroid. To determine whether hydrocortisone was acting directly on the progenitor cell or via an effector cell, its effect on modulating cell populations and stimulating-factor production was studied. Removal of $\mathbf{E}$ rosetting cells and/or adherent cells did not affect the inhibition of eosinophil colony growth or the enhancement of neutrophil colony growth. Furthermore, addition of the potent inhibitor of $T$ cell function, cyclosporin $A$, failed to affect eosinophil colony frequency, suggesting that inhibition of $T$ cell function was an unlikely explanation for the observed hydrocortisone effect. Leukocyte conditioned media (LCM), derived from peripheral blood mononuclear cells incubated with hydrocortisone, was devoid of both neutrophil and eosinophil colony-stimulating activity, whereas a control LCM stimulated both neutrophil and eosinophil proliferation. The data suggest that the observed hydrocortisone effect on granulocyte colony formation is unlikely to be mediated by an intermediary, and that hydrocortisone acts directly on progenitor cells.
\end{abstract}

\section{Introduction}

Glucocorticosteroid therapy results in changes in the numbers of circulating granulocytes. Characteristically, peripheral blood

This paper was presented in part at the 11th Annual Meeting of the International Society for Experimental Hematology, Baltimore, MD, 1982.

Dr. Bjornson is partially supported by a Charles A. King Trust Research Fellowship. Address correspondence to Dr. Bjornson, Department of Hematology/Oncology.

Received for publication 23 January 1984 and in revised form 23 May 1984.

J. Clin. Invest.

(c) The American Society for Clinical Investigation, Inc.

0021-9738/85/09/0924/06 \$1.00

Volume 76, September 1985, 924-929 neutrophilia (1) and eosinopenia are observed (2). In vitro studies have also demonstrated an effect on granulopoiesis by glucocorticosteroids. Golde reported inhibition of murine in vitro colony-forming cells (CFU-C) ${ }^{1}$ by dexamethasone (3) and Zalman et al. (4) confirmed this finding, demonstrating an $18 \%$ decrease in the number of CFU-C when $6 \times 10^{-8} \mathrm{M}$ dexamethasone was incorporated into the culture. Finally, in a study of the effect of hydrocortisone on human CFU-C, Morra et al. (5) found that $1 \mathrm{mg}$ of hydrocortisone added to in vitro cultures decreased the growth of CFU-C by $\sim 24 \%$ (5). Using the Luxol Fast Blue stain, it has been shown that $50 \%$ of the circulating CFU-C (6) and 10-40\% of the bone marrow CFU-C are eosinophilic, with the remainder being neutrophil/macrophage precursors $(7,8)$. To determine whether a differential effect of glucocorticosteroids on eosinophilic and neutrophilic stem cells could be partly responsible for the changes in the levels of mature neutrophils and eosinophils during glucocorticosteroid therapy, we undertook the present study.

Utilizing the double layer soft agar technique, the effect of physiologic to pharmacologic concentrations of hydrocortisone on the proliferation of human neutrophil progenitors and eosinophil progenitors from peripheral blood and bone marrow was examined. The data show a differential effect on the production of these two types of granulocyte colonies. To define the mechanisms by which hydrocortisone may affect eosinophilic proliferation, additional experiments were performed. The effect of progesterone, a structurally similar steroid which in some systems has been shown to block glucocorticoid binding to receptors (9), was studied. T cells have been shown to produce eosinophilopoietic factors (10). Thus, to determine whether the steroids act directly on a progenitor cell or indirectly via an effector cell, whole mononuclear cells, monocyte-depleted nonadherent cells, and T-depleted nonadherent cells were cultured with and without hydrocortisone. To further define the role of T cells, cyclosporin A, a potent immunosuppressive agent, which has been shown to inhibit preferentially early $\mathrm{T}$ cell activation (11), was added to our culture system and the results were compared with those of the hydrocortisone-containing culture. The possibility that hydrocortisone was blocking production of a specific eosinophil colony-stimulating product was examined by preparing leukocyte conditioned media (LCM) from cells cultured with and without hydrocortisone. The data suggest that glucocorticosteroids directly suppress eosinophil colony but not neutrophil colony formation.

\section{Methods}

Venous blood or marrow aspirates were collected in preservative-free heparin from healthy volunteers. Whole mononuclear cells (WMNC)

1. Abbreviations used in this paper: CFU-C, in vitro colony-forming units; CSA, colony-stimulating activity; FCS, fetal calf serum; LCM, leukocyte conditioned medium; MEM, minimal essential medium; NAC, nonadherent cells; WMNC, whole mononuclear cells. 
were separated from the samples on Ficoll-Hypaque gradients. After washing the WMNC three times in alpha minimal essential medium (MEM) containing 20\% fetal calf serum (FCS), the cells were incubated in plastic petri dishes at $37^{\circ} \mathrm{C}$ for $1 \mathrm{~h}$ to remove adherent cells. The nonadherent cells (NAC) were collected and incubated in a fresh petri dish for an additional hour. NAC were mixed with sheep erythrocytes to form E-rosettes. After an overnight incubation, this preparation was subjected to Ficoll-Hypaque separation, and the E-rosette negative cells were collected from the interface.

Preparation of $L C M$. A modification of the method described by Iscove et al. (12) was employed to prepare LCM. WMNC were incubated for $7 \mathrm{~d}$ at $5 \% \mathrm{CO}_{2}, 37^{\circ} \mathrm{C}$, at a concentration of $10^{6} / \mathrm{ml}$ of alpha MEM containing $20 \%$ FCS and $0.5 \mathrm{mM} \beta$-mercaptoethanol. At the end of $7 \mathrm{~d}$, the medium was centrifuged at $1,200 \mathrm{rpm}$ for $15 \mathrm{~min}$. The supernatant was collected and concentrated 12-fold on an Amicon ultrafiltration cell (Diaflo PM 10 membrane). The concentrate was passed through a $0.22-\mu \mathrm{m}$ millipore filter and stored at $0^{\circ} \mathrm{C}$ until further use. Titration revealed maximal colony-stimulating activity (CSA) with 0.1 $\mathrm{ml} \mathrm{LCM} / \mathrm{ml}$ feeder layer. To study the effect of hydrocortisone on colonystimulating factor production, LCM was prepared as described above except $10^{-5} \mathrm{M}$ hydrocortisone was added at the beginning of the incubation. The control LCM contained no added hydrocortisone.

Preparation of cultures. A double layer soft agar culture was employed and cultures were prepared in triplicate. The feeder layer contained 0.1 $\mathrm{ml}$ of LCM in alpha MEM to a final volume of $1 \mathrm{ml}$, containing $15 \%$ FCS and $0.5 \%$ agar. The overlayer in all experiments contained $5 \times 10^{5}$ peripheral blood or $2 \times 10^{5}$ bone marrow NAC in $1 \mathrm{ml}$ of alpha MEM, $15 \% \mathrm{FCS}$, and $0.3 \%$ agar.

Hydrocortisone succinate (courtesy of Upjohn Co., Kalamazoo, MI) was added to the culture overlayers to achieve concentrations of $10^{-5}$, $10^{-6}, 10^{-7}$, and $10^{-8} \mathrm{M}$. Controls contained no added hydrocortisone.
Cyclosporin A (kindly provided by Dr. John B. Harley, National Institutes of Health, Bethesda, MD) was solubilized in absolute ethanol $(1 \mathrm{mg} / \mathrm{ml})$ and incorporated into culture overlayers in amounts ranging from 0.1 to $10 \mu \mathrm{g} / \mathrm{ml}$. Progesterone (Sigma Chemical Co., St. Louis, MO) was solubilized in absolute ethanol and added to cultures in concentrations ranging from $10^{-5}$ to $10^{-9} \mathrm{M}$. To determine progesterone's ability to block the hydrocortisone effect, both hydrocortisone $\left(10^{-5} \mathrm{M}\right)$ and progesterone $\left(10^{-5} \mathrm{M}-10^{-9} \mathrm{M}\right)$ were added to the culture overlayer. Since progesterone and cyclosporin A were both prepared in ethanol, the control cultures for these experiments contained $0.01-0.15 \%$ ethanol.

\section{Results}

Neutrophil colonies were easily distinguished from eosinophil colonies, using the Luxol Fast Blue stain, since neutrophils grow in a loose pattern and their cytoplasm stains a pale blue; in contrast, eosinophil colonies stain a bright aquamarine blue and grow in a tight pattern. The addition of hydrocortisone to the cultures did not affect the characteristic staining patterns nor influence cell morphology. For example, those few eosinophils present in $10^{-5}$ and $10^{-6} \mathrm{M}$ hydrocortisone containing cultures appeared well granulated, and their granules stained the characteristic bright aquamarine blue.

Addition of hydrocortisone to the cultures produced a dosedependent inhibition of peripheral blood eosinophil colony formation (Table I). Bone marrow, which has a lower proportion of eosinophil colony-forming cells, was similarly inhibited (Table II). The frequency of esterase-positive monocyte/macrophage colonies was unaffected by hydrocortisone $(3 \pm 1$ vs. $5 \pm 2 \%)$. The

Table I. Steroid Dose-Response Effect on Granulocyte Colony Formation

\begin{tabular}{|c|c|c|c|c|c|c|c|c|c|c|c|c|c|c|c|}
\hline \multirow[b]{2}{*}{ Experiment } & \multicolumn{3}{|l|}{ Control } & \multicolumn{3}{|l|}{$10^{-8} \mathrm{M}$} & \multicolumn{3}{|l|}{$10^{-7} \mathrm{M}$} & \multicolumn{3}{|l|}{$10^{-6} \mathrm{M}$} & \multicolumn{3}{|l|}{$10^{-5} \mathrm{M}$} \\
\hline & $\begin{array}{l}\text { Total no. } \\
\text { colonies }\end{array}$ & $\frac{\text { Neut }}{\text { Eos }}$ & Eos & $\begin{array}{l}\text { Total no. } \\
\text { colonies }\end{array}$ & $\frac{\text { Neut }}{\text { Eos }}$ & Eos & $\begin{array}{l}\text { Total no. } \\
\text { colonies }\end{array}$ & $\frac{\text { Neut }}{\text { Eos }}$ & Eos & $\begin{array}{l}\text { Total no. } \\
\text { colonies }\end{array}$ & $\frac{\text { Neut }}{\text { Eos }}$ & Eos & $\begin{array}{l}\text { Total no. } \\
\text { colonies }\end{array}$ & $\frac{\text { Neut }}{\text { Eos }}$ & Eos \\
\hline & & & $\%$ & & & $\%$ & & & $\%$ & & & $\%$ & & & $\%$ \\
\hline 1 & 22 & $\frac{12}{10}$ & 48 & 28 & $\frac{15}{13}$ & 45 & 33 & $\frac{17}{16}$ & 49 & 25 & $\frac{22}{3}$ & 13 & 25 & $\frac{24}{1}$ & 5 \\
\hline 2 & 22 & $\frac{9}{13}$ & 59 & 10 & $\frac{5}{5}$ & 46 & 16 & $\frac{11}{4}$ & 25 & 18 & $\frac{16}{1}$ & 4 & 16 & $\frac{15}{1}$ & 2 \\
\hline 3 & 94 & $\frac{45}{47}$ & 50 & 83 & $\frac{45}{37}$ & 45 & 99 & $\frac{56}{42}$ & 42 & 64 & $\frac{54}{10}$ & 16 & 60 & $\frac{55}{5}$ & 8 \\
\hline 4 & 32 & $\frac{20}{11}$ & 34 & 25 & $\frac{17}{7}$ & 28 & 16 & $\frac{14}{2}$ & 12 & 26 & $\frac{21}{5}$ & 18 & 16 & $\frac{15}{1}$ & 4 \\
\hline 5 & 17 & $\frac{7}{9}$ & 53 & 22 & $\frac{13}{9}$ & 40 & 15 & $\frac{12}{3}$ & 18 & 17 & $\frac{16}{1}$ & 4 & 14 & $\frac{13}{1}$ & 0 \\
\hline Mean & 37 & & 49 & 34 & & 41 & 36 & & 29 & 30 & & 11 & 26 & & 4 \\
\hline SEM & 14 & & 4 & 13 & & 3 & 16 & & 7 & 9 & & 3 & 9 & & 1 \\
\hline $\begin{array}{l}P \text { (Control vs. } \\
\text { dose of hydro- } \\
\text { cortisone) }\end{array}$ & & & & & & NS & & & 0.05 & & & 0.01 & & & 0.01 \\
\hline $\begin{array}{l}\text { \% Change from } \\
\text { control }\end{array}$ & & & & -9.37 & & -16.39 & -3.64 & & -40.16 & -18.80 & & -77.46 & -30 & & -92.21 \\
\hline
\end{tabular}

The effect of hydrocortisone succinate on the total number of colonies (neutrophil, eosinophil, and mixed), and the proportion that were eosinophil. The values were derived from the colonies generated by $5 \times 10^{5}$ peripheral blood NAC and represent the mean of triplicate plates. The percentage of eosinophil colonies was analyzed using one-way analysis of variance followed by Newman-Keuls test. Neut, neutrophil; Eos, eosinophil. 
Table II. Effect of Hydrocortisone on Bone Marrow Granulocyte Colony Formation

\begin{tabular}{|c|c|c|c|c|c|c|}
\hline \multirow[b]{2}{*}{$\begin{array}{l}\text { Experi- } \\
\text { ment }\end{array}$} & \multicolumn{3}{|l|}{ Control } & \multicolumn{3}{|c|}{$10^{-5} \mathrm{M}$ Hydrocortisone } \\
\hline & $\begin{array}{l}\text { Total } \\
\text { no. } \\
\text { colonies }\end{array}$ & $\frac{\text { Neut }}{\text { Eos }}$ & $\begin{array}{l}\text { Percentage } \\
\text { Eos }\end{array}$ & $\begin{array}{l}\text { Total } \\
\text { no. } \\
\text { colonies }\end{array}$ & $\frac{\text { Neut }}{\text { Eos }}$ & $\begin{array}{l}\text { Percentage } \\
\text { Eos }\end{array}$ \\
\hline & & 178 & & & 204 & \\
\hline \multirow[t]{2}{*}{1} & 220 & $\overline{42}$ & 19 & 204 & $\overline{0}$ & 0 \\
\hline & & $\underline{16}$ & & & $\underline{12}$ & \\
\hline \multirow[t]{2}{*}{2} & 24 & $\overline{8}$ & 33 & 12 & 0 & 0 \\
\hline & & $\underline{42}$ & & & 44 & 0 \\
\hline \multirow[t]{2}{*}{3} & 73 & $\overline{31}$ & 43 & 44 & 0 & \\
\hline & & $\underline{50}$ & & & $\underline{51}$ & \\
\hline 4 & 57 & $\overline{7}$ & 12 & 51 & $\overline{0}$ & 0 \\
\hline
\end{tabular}

The values were derived from the colonies generated by $2 \times 10^{5}$ marrow NAC and represent the mean of triplicate plates. $P=0.028$ by the Mann-Whitney U test. Neut, neutrophil; Eos, eosinophil.

growth of peripheral blood neutrophil colonies increased with the addition of hydrocortisone (Fig. 1). When the effect of the addition of $10^{-8} \mathrm{M}$ hydrocortisone was compared with control, the total number of colonies decreased by $9.4 \%$ and the proportion that were eosinophil colonies decreased by $16.4 \%$. With the addition of $10^{-7} \mathrm{M}$ hydrocortisone, the total number of colonies decreased by $3.6 \%$, but the proportion that were eosinophilic was $40.2 \%$ less than control $(P<0.05)$. In the presence of pharmacologic concentrations of hydrocortisone $\left(10^{-6} \mathrm{M}\right)$, the total number of colonies decreased by $18.8 \%$, while the decrease in the number of eosinophil colonies was $77.5 \%(P$ $<0.01$ ). Finally, at $10^{-5} \mathrm{M}$, the total number of colonies decreased by $30 \%$, but the number of eosinophil colonies decreased by $92.2 \%(P<0.01)$. The number of mixed neutrophil eosinophil colonies observed ranged from 0 to $2 \%$ and was not affected by

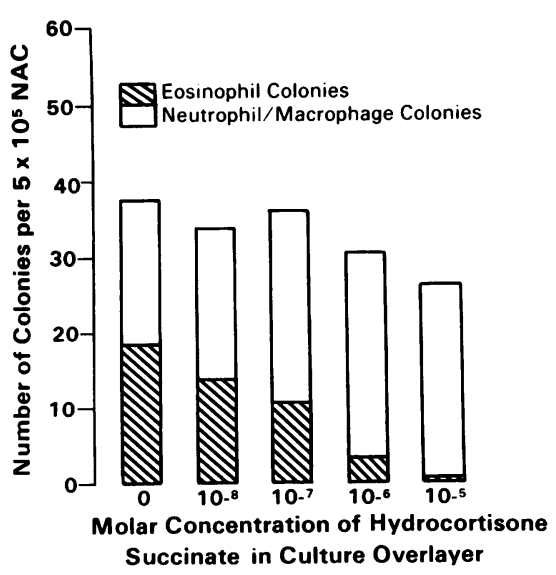

Figure 1. The effect of hydrocortisone succinate on the number of eosinophil and neutrophil colonies. The number of eosinophil colonies (diagonally lined bars) and neutrophil/macrophage colonies (clear bars) generated by $5 \times 10^{5} \mathrm{NAC}$ were quantitated as described in Methods. The values are the means for the five experiments listed in Table I. the addition of hydrocortisone. For percentage of eosinophil colonies, the five groups (control, $10^{-8}, 10^{-7}, 10^{-6}$, and $10^{-5} \mathrm{M}$ ) were compared using one-way analysis of variance followed by Newman-Keuls test.

When hydrocortisone was added to cultures after plating, a progressive loss of ability to decrease eosinophil colony formation was observed over $24 \mathrm{~h}$ (Fig. 2). To determine the reversibility of the hydrocortisone effect, suspensions of peripheral blood NAC were exposed to either $10^{-5} \mathrm{M}$ hydrocortisone or medium alone for $24 \mathrm{~h}$, then the cells were washed three times and plated for colony growth. Hydrocortisone had no significant effect on viable cell yields from control and steroid-treated suspensions. The percentage of trypan blue-excluded cells was 90 and $95 \%$ of the starting number, respectively. 24-h exposure to hydrocortisone diminished the frequency of eosinophil colonies in seven of seven experiments $(P<0.009$, Wilcoxon Signed Rank test); however, the diminution was less than that observed with continuous hydrocortisone exposure, suggesting partial reversibility (Table III).

A sample experiment showing the effect of hydrocortisone on eosinophil colony formation by peripheral blood samples depleted of various cell populations is summarized in Table IV. This data is analyzed using a two-way analysis of variance. Results from three separate experiments show that when WMNC were adherent-cell and T-cell depleted, the percentage of eosinophil colonies increased in both control and $10^{-5} \mathrm{M}$ hydrocortisone-treated cultures. Adherent cell depletion alone was without effect, with an eosinophil colony frequency of $49 \pm 6 \%$. The same degree of inhibition of eosinophil colony growth was observed in the WMNC, NAC, and T cell-depleted NAC populations cultured with $10^{-5} \mathrm{M}$ hydrocortisone $(P<0.001)$.

To further examine the possibility that hydrocortisone was specifically suppressing eosinophil CSA production by accessory cell populations, we studied the effect of LCMs prepared with and without hydrocortisone $\left(10^{-5} \mathrm{M}\right)$ on peripheral blood mononuclear cell eosinophil colony frequency. Hydrocortisonecontaining LCMs did not stimulate colony formation above the background level of $<4$ colonies $/ 5 \times 10^{5}$ cells, whereas the con-

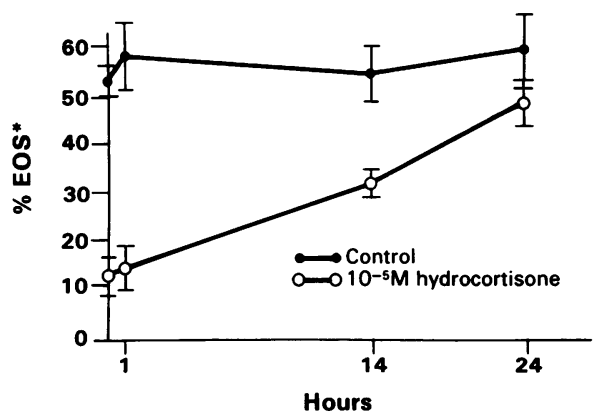

Figure 2. Time course of steroid effect on granulocyte proliferation. A representative experiment demonstrating the effect of hydrocortisone on the frequency of eosinophil colonies when hydrocortisone was added at various times during culture incubation. The experiment was performed as described in Methods except for the addition of hydrocortisone succinate to gelled overlayers in contrast to experiments in which it was mixed with cells before plating. The values expressed are the mean of triplicate plates. Solid circles represent control values and open circles the hydrocortisone-treated culture values. The control does not change over time while, there is inhibition of eosinophil proliferation when hydrocortisone is added during the initial hours of culture incubation. 
Table III. Partial Reversibility of the Hydrocortisone Effect

\begin{tabular}{|c|c|c|c|c|}
\hline \multirow[b]{2}{*}{ Experiment } & \multicolumn{2}{|l|}{ Control NAC } & \multicolumn{2}{|c|}{$\begin{array}{l}\text { NAC incubated for } 24 \mathrm{~h} \text { with } \\
10^{-5} \mathrm{M} \text { hydrocortisone succinate }\end{array}$} \\
\hline & Total colonies & Percent Eos & Total colonies & Percent Eos \\
\hline 1 & 25 & 44 & 8 & 30 \\
\hline 2 & 20 & 48 & 13 & 0 \\
\hline 3 & 85 & 69 & 54 & 2 \\
\hline 4 & 30 & 41 & 15 & 22 \\
\hline 5 & 12 & 44 & 44 & 32 \\
\hline 6 & 29 & 35 & 31 & 22 \\
\hline 7 & 41 & 49 & 25 & 18 \\
\hline
\end{tabular}

The values represent the mean of triplicate plates and were derived from the colonies generated by $5 \times 10^{5}$ peripheral blood NAC, incubated $24 \mathrm{~h}$, and then washed in fresh media. Eos, eosinophil. $P$ $=0.009$ by Wilcoxon Signed Rank Test.

trol LCMs produced a mean of $37 \pm 7$ colonies $(n=3)$. To determine if the inactivity of LCM prepared with hydrocortisone was due to the fact that hydrocortisone remained in the LCM, $10^{-5} \mathrm{M}$ hydrocortisone succinate was added to harvested LCM that was screened and known to contain CSA. The LCM produced 72 colonies, of which $64 \%$ were eosinophils. When hydrocortisone was added, 44 colonies were produced, of which $10 \%$ were eosinophils $(n=2)$. Thus, the hydrocortisone added to the LCM did not inhibit neutrophil proliferation, but did suppress eosinophil proliferation.

The addition of $10^{-5}-10^{-9} \mathrm{M}$ progesterone, a steroid analogue, which in some systems has been shown to block glucocorticosteroid binding to receptors (9), inhibited colony growth at concentrations of $10^{-7} \mathrm{M}$ or greater, but did not alter the ratio of neutrophil to eosinophil colonies. Furthermore, progesterone did not block the effect of hydrocortisone when both steroids were added to the culture.

To further examine the role of accessory cell populations in hydrocortisone-mediated inhibition of eosinophil colony formation, the effect of cyclosporin A, a drug shown to block early $T$ cell activation (11), was tested. The addition of cyclosporin A to the cultures at concentrations of $0.1-10 \mu \mathrm{g} / \mathrm{ml}$, failed to

Table IV. Effect of Hydrocortisone on the Percentage of Eosinophil Colonies Cultured from Peripheral Blood Depleted of Adherent and E-Rosetting Cells

\begin{tabular}{llc}
\hline & \multicolumn{2}{l}{ Percentage of eosinophil colonies } \\
\cline { 2 - 3 } Cell populations & Control & $10^{-5}$ M Hydrocortisone* \\
\hline WMNC & $55 \pm 8$ & $10 \pm 3$ \\
NAC & $49 \pm 6$ & $5 \pm 3$ \\
T-Depleted NAC $\ddagger$ & $75 \pm 5$ & $12 \pm 5$
\end{tabular}

The data represent the mean and standard error of percent eosinophil colony formation from three separate experiments. The number of colonies per $5 \times 10^{5} \mathrm{WMNC}$ ranged from 23 to 78 . Two way analysis of variance: * Control vs. $10^{-5} \mathrm{M}$ hydrocortisone $(P<0.001)$ (for each type of cell population). $\ddagger$ T-depleted NAC vs. WMNC $(P<0.05$ ) (for both control and $10^{-5} \mathrm{M}$ hydrocortisone).
Table V. Effect of Cyclosporin A on Total Colony Number and Percentage Eosinophil

\begin{tabular}{lllll}
\hline & & \multicolumn{3}{l}{ Cyclosporin concentrations } \\
\cline { 3 - 5 } Experiment & Control & $10 \mu \mathrm{g} / \mathrm{ml}$ & $1 \mu \mathrm{g} / \mathrm{ml}$ & $0.1 \mu \mathrm{g} / \mathrm{ml}$ \\
\hline 1 & $39(46)^{*}$ & $2(50)$ & $24(36)$ & $21(48)$ \\
2 & $41(45)$ & $1(0)$ & $16(33)$ & $29(38)$
\end{tabular}

* Total number of colonies (percent eosinophil colonies) generated by $5 \times 10^{5}$ peripheral blood NAC. The values are the mean of triplicate plates.

affect the frequency of eosinophil colony formation despite a $95 \%$ decrease in colony growth at $10 \mu \mathrm{g} / \mathrm{ml}$ (Table V).

\section{Discussion}

In vitro (13) and in vivo (14) studies have shown a differential control of eosinophil and neutrophil production. Almost all sources of CSA contain neutrophil/monocyte as well as eosinophil CSA, and attempts have been made to separate these factors. Using hydrophobic chromatography, Nicola et al. (15) have prepared $\beta$-fraction human placental conditioned media that stimulates proliferation of only neutrophil/monocyte colonies. The $\alpha$-fraction that contains eosinophil CSA also contains neutrophil/monocyte CSA. Thus, it is not yet clear whether eosinophil and neutrophil/monocyte CSA are part of the same molecule, or if improved separation techniques will prove they are distinct factors. Potentially important physiologic and pharmacologic regulators of eosinophil production and distribution are glucocorticosteroid hormones. Although many workers have shown that corticosteroids inhibit in vitro growth of CFU-C, none have examined its effect on the selective growth of neutrophil and eosinophil colonies. Since eosinophilic CFU-C can account for up to $40 \%$ of bone marrow-derived granulocyte colonies (8), and up to $50 \%$ of peripheral blood colonies (6), selective suppression of eosinophil colonies by corticosteroids could mask an actual increase in neutrophil colonies. Suggestive evidence for this is provided by Joyce and Chervenick (16), who found enhanced recovery of granulopoiesis when corticosteroids were given to mice treated with cyclophosphamide. Similarly, Niskanen and Squires (9) found enhanced diffusion chamber granulocyte colony formation in mice treated with corticosteroids. We examined the in vitro effect of glucocorticosteroids on human eosinophil and neutrophil colony formation, and demonstrated that whereas corticosteroids suppress eosinophil colony formation, they actually enhance neutrophil colony formation.

When corticosteroids were added to cultures of peripheral blood or marrow, a dose-dependent inhibition of eosinophil colony formation was observed, with $>95 \%$ inhibition observed at $10^{-5} \mathrm{M}$ hydrocortisone. On the other hand, the actual number of neutrophil colonies per $10^{5}$ cells cultured increased by $31 \%$ when $10^{-5} \mathrm{M}$ hydrocortisone was added to the cultures $(P$ $<0.01)$. Therefore, although hydrocortisone decreased the total number of granulocyte colonies, this decrease was the summation of an increase in neutrophil colony formation and a profound decrease in eosinophil colony growth. The enhancement of neutrophil colony growth by hydrocortisone was greater in peripheral blood cultures than in marrow cultures. This suggests that the peripheral blood contains a greater number of less differentiation- 
restricted (bipotent) granulocyte progenitors, which are capable of being induced to differentiate along either a neutrophil or eosinophil line.

In order for corticosteroids to affect neutrophil and eosinophil proliferation, they had to be added within $24 \mathrm{~h}$ of initiation of the cultures (Fig. 2). If hydrocortisone was added after $24 \mathrm{~h}$, the inhibition of eosinophil colony formation was abrogated. These same experiments demonstrated that hydrocortisone did not exert its effect by lysis of eosinophil colonies, since plates to which hydrocortisone had been added after $24 \mathrm{~h}$ contained well-granulated normal appearing eosinophil colonies, in numbers only slightly lower than those in the control cultures. On the other hand, when suspension cultures of peripheral blood NAC were exposed to hydrocortisone for $24 \mathrm{~h}$, only a partial inhibition of subsequent eosinophil colony formation resulted, suggesting there is partial reversibility of the effect. It is also possible that nonaffected cells escaped the drug effect by not entering a proliferative phase, but there is no evidence to support this mechanism.

Niskanen and Squires (9) reported that the stimulatory effect of corticosteroids on granulocyte proliferation in diffusion chambers was blocked by progesterone, and the authors suggested that the mechanism of this effect was competition for the steroid receptor. However, since progesterone is capable of inhibiting granulopoiesis in murine systems, the observed blocking effect may have been due to direct inhibition of granulopoiesis as observed by others (17). We examined the effect of progesterone when added alone or in combination with hydrocortisone on neutrophil and eosinophil colony formation. Progesterone, in concentrations as low as $10^{-8} \mathrm{M}$, inhibited both eosinophil and neutrophil colony formation by $40 \%$. However, when added in combination with hydrocortisone, it failed to alter the hydrocortisone effect, showing that in this system progesterone does not block the hydrocortisone effect.

We examined whether hydrocortisone suppresses eosinophil colony formation by its effect on potential modulating cell populations. The data suggested that the hydrocortisone effect was not detectably mediated by accessory cell populations, and may have been a direct one on the granulocyte progenitor. Removal of E-rosetting cells and or adherent cells did not affect the inhibition of eosinophil colony growth or the enhancement of neutrophil colony growth by hydrocortisone. When both adherent and E-rosetting cells were removed, hydrocortisone decreased eosinophil growth by $83 \%$ and increased neutrophil colony growth by $56 \%$. Furthermore, addition of cyclosporin A, a potent inhibitor of $\mathrm{T}$ cell function, failed to affect eosinophil colony frequency when added alone, and when cyclosporin A was added to cultures containing $10^{-5} \mathrm{M}$ hydrocortisone it failed to alter the previously described response to hydrocortisone.

Metcalf (18) has reported that when mice are treated with cortisone acetate, the level of measureable colony-stimulating factor in the serum falls sharply. Similarly, Ralph et al. (19) showed that addition of $10^{-4} \mathrm{M}$ hydrocortisone to macrophage cell lines completely inhibited lipopolysaccharide-induced production of myeloid CSA; however, it had no effect on constitutive production of myeloid CSA (19). To determine whether the inhibition of eosinophil colony formation we observed with hydrocortisone was mediated by a differential suppression of eosinophil CSA by accessory cells, conditioned media was prepared from whole blood mononuclear cells incubated with $10^{-5} \mathrm{M}$ hydrocortisone and tested for its ability to stimulate eosinophil and neutrophil colony formation by nonadherent blood and marrow mononuclear cells. As previously observed by others (18), preparations of LCM derived from cells incubated with hydrocortisone were devoid of both neutrophil and eosinophil CSA, whereas control LCMs stimulated $18 \pm 4$ eosinophil and $20 \pm 6$ neutrophil colonies per $5 \times 10^{5}$ peripheral blood NAC. These data indicate that the observed hydrocortisone effect on granulocyte colony formation was unlikely to be mediated by an extracellular humor.

On the basis of cluster transfer experiments (20), and the partial separability of eosinophil and neutrophil progenitor cells by velocity sedimentation gradients, it has been postulated that eosinophils constitute a separate lineage with their own progenitor cells. Although nothing in the present data contradicts this hypothesis, it does suggest an alternative explanation for the suppression of eosinophil colonies with the simultaneous increase in neutrophils colonies. It is possible that some progenitor cells are bipotent, and can switch from eosinophil to neutrophil commitment by the modulating action of hydrocortisone. However, the nearly complete suppression of eosinophil colony formation and the overall decrease in total granulocyte colony numbers in the presence of hydrocortisone indicates that hydrocortisone inhibits proliferation of those progenitor cells solely committed to eosinophilopoiesis. A possible mechanism for the observed hydrocortisone effect is corticosteroid-induced modulation of cell surface receptors for regulatory molecules. Studies in other systems have shown that glucocorticosteroids are capable of altering the number of cell surface receptors for various trophic substances (21-23). For example, treatment of human subjects with glucocorticosteroids decreased the number of adrenergic receptor sites on their mononuclear cells while increasing the number on their neutrophils (23). Such a differential effect of glucocorticosteroids on eosinophil progenitors vs. neutrophil progenitors may account for the decreased production of eosinophils and increased proliferation of neutrophils.

\section{Acknowledgments}

The authors wish to thank Drs. Jack W. Singer and Alfred I. Tauber for their assistance and advice, and Ms. Ann Marie Happnie for careful preparation of the manuscript.

This work was supported by grants IN-97G from the American Cancer Society and RR-05569-17 from the Biomedical Research Support Program of Boston City Hospital. Dr. Bjornson is supported by a National Institutes of Health New Investigator Research Award (20398).

\section{References}

1. Bishop, C. R., J. W. Athens, D. R. Boggs, H. R. Warner, G. E. Cartwright, and M. M. Wintrobe. 1968. Leukokinetic studies. XIII. A non-steady state kinetic evaluation of the mechanism of cortisone-induced granulocytosis. J. Clin. Invest. 47:249-260.

2. Kellegren, J. H., and O. Janus. 1951. The eosinopenic response to cortisone and ACTH in normal subjects. Br. Med. J. 2:1183-1187.

3. Golde, D. W., N. Bersh, S. G. Quan, and M. J. Cline. 1976. Inhibition of murine granulopoiesis in vitro by dexamethasone. Am. J. Hematol. 1:369-373.

4. Zalman, F., M. W. Maloney, and H. M. Patt. 1979. Differential response of early erythropoietic and granulopoietic progenitors to dexamethasone and cortisone. J. Exp. Med. 149:67-72.

5. Morra, L., A. Ponassi, G. B. Parodi, G. Caristo, P. Bruzzi, and C. Sacchetti. 1981. Mobilization of colony-forming cells (CFU-C) into the peripheral blood of man by hydrocortisone. Biomedicine (Paris). 35:8790.

6. Bjornson, B. H., J. Andre-Schwartz, and J. F. Desforges. 1982. In 
vitro culture of circulating CFU-eos from normal donors. Exp. Hematol. 10:271-276.

7. Dresch, C., G. R. Johnson, and D. Metcalf. 1977. Eosinophil colony formation in semisolid cultures of human bone marrow cells. Blood. 49: 834-844.

8. Verma, D. S., G. Spitzer, A. R. Zander, R. Fisher, K. B. McCredie, and K. Dicke. 1980. The myeloid progenitor cells: a parallel study of subpopulations in human marrow and peripheral blood. Exp. Hematol. 8:32-43.

9. Niskanen, E., and J. Squires. 1981. Corticosteroid effect on murine hemopoietic precursor cells in vivo. Blood. 57:1138-1139.

10. Walls, R. S. 1977. Eosinophil response to alum adjuvants: involvement of T-cells in nonantigen-dependent mechanisms. Proc. Soc. Exp. Biol. Med. 156:431-435.

11. Wiesinger, D., and J. F. Borel. 1979. Studies on the mechanism of action of Cyclosporin A. Immunobiology. 156:454.

12. Iscove, N. N., J. S. Senn, J. E. Till, and E. A. McCulloch. 1971. Colony formation by normal and leukemic human marrow cells in culture. Effect of conditioned medium from human leukocytes. Blood. 37: $1-5$.

13. Bartelmez, S. H., W. H. Dodge, and D. A. Bass. 1980. Differential regulation of spleen cell-mediated eosinophil and neutrophil/macrophage production. Blood. 55:489-493.

14. Beeson, P. B., and D. A. Bass. 1977. The Eosinophil. W. B. Saunders \& Co., Philadelphia. 79-91.

15. Nicola, N. A., D. Metcalf, G. R. Johnson, and A. W. Burgess.
1979. Separation of functionally-distinct human granulocyte-macrophage colony stimulating factors. Blood. 54:614-627.

16. Joyce, R. A., P. A. Chervenick. 1977. Corticosteroid effect on granulopoiesis in mice after cyclophosphamide. J. Clin. Invest. 60:277.

17. Singer, J. W., A. I. Samuels, and J. W. Adamson. 1976. Steroids and hematopoiesis. J. Cell. Physiol. 88:127-133.

18. Metcalf, D. 1969. Cortisone action on serum colony stimulating factor and bone marrow in vitro colony forming cells. Proc. Soc. Exp. Biol. Med. 132:391-394.

19. Ralph, P., M. Ito, H. E. Broxmyer, and I. Nakoinz. 1978. Corticosteroids block newly induced but not constitutive functions of macrophage cell lines: myeloid colony stimulating activity production, latex phagocytosis, and antibody-dependent lysis of rbc and tumor targets. $J$. Immunol. 121:300-303.

20. Dao, C., D. Metcalf, and E. R. Bilski-Pasqui. 1977. Eosinophil and neutrophil colony forming cells in culture. Blood. 50:833-839.

21. Wong, G. L., B. P. Lukert, and J. S. Adams. 1980. Glucocorticosteroids increase osteoblast-like bone cell response to $1,25(\mathrm{OH})_{2} \mathrm{D}_{3}$. Nature (Lond.). 285-254.

22. Baker, J. B., G. S. Barsh, D. H. Carney, and D. D. Cunninghan. 1978. Dexamethasone modulates binding and action of epidermal growth factor in serum-free cell culture. Proc. Natl. Acad. Sci. USA. 75: $1882-1886$.

23. Motulsky, H. J., and P. A. Insel. 1982. Adrenergic receptors in man: direct identification, physiologic regulation and clinical alterations. N. Engl. J. Med. 307:18-29. 